Z Rheumatol 2010 $69: 772-773$

DOI 10.1007/s00393-010-0638-9

(c) Springer-Verlag 2010

A. Zink ${ }^{1} \cdot$ M. Schneider ${ }^{2}$

${ }^{1}$ Forschungsbereich Epidemiologie, Deutsches Rheuma-Forschungszentrum Berlin und Abteilung für Rheumatologie und Klinische Immunologie,

Charité-Universitätsmedizin Berlin

${ }^{2}$ Klinik für Endokrinologie, Diabetologie und Rheumatologie,

Heinrich-Heine-Universität Düsseldorf

\title{
10 Jahre Behandlung mit TNF-Inhibitoren - Sind wir auf der sicheren Seite?
}

Gruppen, die nie in eine klinische Prüfung eingeschlossen worden wären, beurteilen zu können, wurden vor rund 10 Jahren in verschiedenen europäischen Ländern unabhängige, von verschiedenen Pharmafirmen gesponserte, Biologika-Register eingerichtet, darunter das deutsche Register RABBIT. In zunehmendem Maße sind die Register nun in der Lage, zumindest vorläufige Antworten auf diese komplexen Fragen zu geben.

\section{(2) Register beurteilen die langfristige Sicherheit der Biologika und das Sicherheitsprofil in speziellen Gruppen}

In diesem Heft wird die Datenlage zu drei relevanten Problemkomplexen dargestellt. Dabei sind die Register eine wichtige, aber nicht die einzige Datengrundlage.

Johan Askling vom schwedischen Biologika-Register ARTIS hat, ausgehend von relevanten klinischen Situationen, eine Übersicht über das aktuelle Wissen zum Malignomrisiko aus den verschiedenen Registern erstellt, und zwar sowohl im Hinblick auf neue Tumoren als auch im Hinblick auf das Risiko, ein Rezidiv eines früheren Tumors zu erleiden. Im Hinblick auf das globale Risiko für inzidente Tumoren können wir inzwischen recht sicher sein, die Patienten keinem hohen zusätzlichen Ri- siko auszusetzen. Eine gewisse Risikoerhöhung ist aber bei Hautkrebs anzunehmen. Widersprüchliche Ergebnisse gibt es für das Risiko eines Tumorrezidivs. Während das deutsche Register eine leichte - und insignifikante - Risikoerhöhung gesehen hat, war das Risiko im britischen Register unter TNF-Inhibitoren sogar etwas verringert gegenüber der DMARD-Gruppe. Ein wesentlicher Unterschied zwischen beiden Registern war die Zeitspanne zwischen Tumorerkrankung und Therapiebeginn mit einem TNF-Inhibitor: Einen Therapiebeginn mit einem TNF-Inhibitor innerhalb von 10 oder weniger Jahren nach der Tumordiagnose hatten im britischen Register $42 \%$ der Patienten, im deutschen hingegen $77 \%$. In Deutschland wurden also mehr Patienten innerhalb eines Zeitfensters behandelt, in dem die Patienten ohnehin einem erhöhten Risiko eines Rezidivs ausgesetzt sind. Die Daten sprechen dafür, die Zeitspanne zwischen Tumordiagnose und Therapiebeginn mit einem TNF-Blocker nicht noch weiter zu verkürzen.

Rebekka Fischer-Betz hat den aktuellen Literaturstand aus Registern und Einzelfallberichten zu dem komplexen Thema „Therapie vor und in der Schwangerschaft" zusammengetragen. Auch wenn die Sorge, in größerem Umfang schwerwiegende Fehlbildungen wie das VACTERL-Syndrom auszulösen, inzwischen 
wenig begründet scheint und keine Hinweise auf eine teratogene Wirkung in den Registern gefunden wurden, sind trotz internationaler Beobachtung und Zusammenarbeit die Zahlen noch zu klein, um eine generelle Entwarnung für die Behandlung in der Schwangerschaft zu geben. Zumindest gibt es keine Hinweise, dass eine Therapie bis zur Konzeption mit einem erhöhten Risiko verbunden ist. Umgekehrt führt ja oftmals die Kontrolle der Krankheitsaktivität erst dazu, dass eine Schwangerschaft eintritt. Es bleibt aber derzeit bei der Empfehlung, bei Kenntnis der Schwangerschaft die Therapie abzusetzen. Alle hiervon abweichenden Therapiestrategien, die bei hoch aktivem Verlauf notwendig werden können, müssen nach ausführlicher Patientenaufklärung zwischen Arzt und Patientin abgewogen und letztlich auch von diesen verantwortet werden.

Eine systematische Übersicht zum „finalen Outcome" Tod legt Oliver Sander vor. Dabei wird u. a. deutlich, wie komplex die Antwort auf eine einfache Frage: „Erhöhen oder senken Biologika das Mortalitätsrisiko?" sein kann. Die schon für Methotrexat vor vielen Jahren von der Arbeitsgruppe um Rolf Rau gezeigte Tatsache, dass eine erfolgreiche, rasch eintretende Senkung der Krankheitsaktivität das Sterberisiko langfristig und deutlich senkt, scheint auch für die TNF-Inhibitoren zu gelten. Wenn es mit konventioneller Therapie nicht gelingt, dieses Ziel zu erreichen, die Patienten aber auf Biologika ansprechen, dann bietet diese Therapie einen Überlebensvorteil. Dies gilt auch für einzelne Todesursachen wie Herzinfarkt oder Lymphome.

Eine thematisch zu den Übersichtsarbeiten passende Originalarbeit von Lotta Gäwert hat anhand der Daten des RABBIT-Registers untersucht, wie gut Angaben von Ärzten und Patienten zu schwerwiegenden unerwünschten Ereignissen übereinstimmen, wenn man den Patienten als Goldstandard nimmt. Die Frage ist also: Welche Beschwerden und Symptome beobachten und berichten Patienten, die vom Arzt nicht berichtet oder nicht der Therapie zugerechnet werden? Diese Frage ist relevant im Hinblick auf die Patientenführung, denn nur ein Patient, der Risiken und Nebenwirkungen der
Therapie korrekt einschätzen kann, wird auch die notwendige Therapietreue aufweisen.

Wir wünschen anregende Lektüre.

Ihre

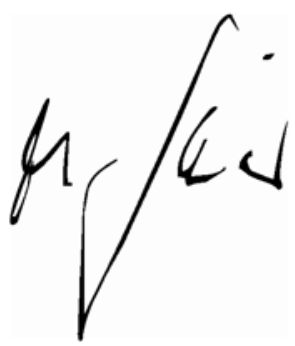

Matthias Schneider

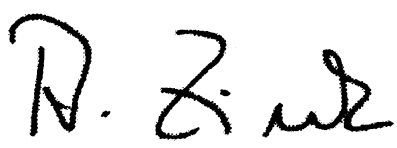

Angela Zink

\section{Korrespondenzadresse}

Prof. Dr. A. Zink

Forschungsbereich Epidemiologie, Deutsches Rheuma-Forschungszentrum Berlin und Abteilung für Rheumatologie und Klinische Immunologie, Charité-Universitätsmedizin Berlin Charitéplatz 1, 10117 Berlin zink@drfz.de

\section{Fiebersyndrome und Autoimmunität bei Kindern im Fokus Dresdner Forscher}

Der neu gegründete interdisziplinäre Forschungsverbund 249 „Defekte des angeborenen Immunsystems bei autoinflammatorischen und autoimmunologischen Erkrankungen" will die Ursachen von pathologischen Entzündungsreaktionen (Fiebersyndrome) und Autoimmunität (Rheuma, Lupus erythematodes) ergründen. Die DFG-geförderte Forschergruppe will auf diese Weise die Grundlagen für ein besseres Verständnis des Immunsystems und die Entwicklung neuer therapeutischer Ansätze schaffen. Ein Schwerpunkt liegt auf einer engeren Verzahnung der klinisch orientierten Patientenforschung mit der Grundlagenforschung. Die biomedizinische wissenschaftliche Nachwuchs soll ebenso schwerpunktmäßig gefördert werden. Zukünftig entstehen eine Professorenstelle und 15 neue Stellen für wissenschaftliche und technische Mitarbeiter. Mit der Einrichtung der ersten Klinischen Forschergruppe der DFG in Dresden wird damit ein wichtiger Beitrag zur wissenschaftlichen Exzellenz und Vielfalt der klinischen Forschung an der Medizinischen Fakultät der TU Dresden geleistet.

Quelle: Universitätsklinikum Carl Gustav Carus Dresden, www.uniklinikum-dresden.de 